International Journal of Engineering \& Technology, $7(2.25)(2018) 43-47$
SPC
International Journal of Engineering \& Technology
Website: www.sciencepubco.com/index.php/IJET
Research paper

\title{
IOT based $\mathrm{e}$ - health care system
}

\section{R. Chandrasekaran ${ }^{1}$, Syed Uzma Farheen ${ }^{2}$, R. J.Hemalatha ${ }^{1 *}$, Bincy Babu ${ }^{1}$, Josephin Arockiya Dhivya ${ }^{1}$, T. R. Thamizhvani $^{1}$}

\author{
${ }^{I}$ Assistant professor Department of Biomedical Engineering, Vels Institute of Science, Technology and Advanced Studies \\ ${ }^{2}$ Student, Department of Biomedical Engineering, Vels Institute of Science, Technology and Advanced Studies \\ *Corresponding author E-mail: hodbiomedical@velsuniv.ac.in
}

\begin{abstract}
One of the most important technological evolutions of our time is CLOUD COMPUTING, which describes the web computing power to store and process the information. The evolution and advancements are swiftly increasing in remote monitoring and Telemedicine. This paper aims at transmitting the physiological parameters of the subject to the private cloud called Thing Speak, an IOT based Sensor monitoring system. The physiological parameters are sent to the cloud via ESP8266 (IOT device). The cloud computing helps the physician to be connected with the patient's data and it is helpful in monitoring the patients at any time through internet.
\end{abstract}

Keywords: IOT; ESP8266; Thing Speak.

\section{Introduction}

Good health is a tremendous gift by god. To monitor patient continuously in critical care, good health care system is a fundamental essential. In the era of rapid improvement of technology, every system is reorganized into 'smart'. As per the latest research, it is said that, by $2019,87 \%$ of health care organizations will have adopted IoT technology. The IoT provides appropriate solutions for a wide range of applications such as smart cities, traffic congestion, waste management, structural health, security, emergency services, logistics, retails, industrial control, and health care . Now a days, Telemedicine has wide growth and has evolved as one of the far-reaching reaseach area. Therefore, healthcare is the most forward looking aspect, as it is the most critical essential in case of any light to chronic problem. Due to the motivational development of android applications, using the merging technology IoT, this approach might be a significant one. In, this paper we propose pulse rate sensor, ECG sensor, temperature sensor and the respiration transducer, from which the sensors and transducer data is sent to the gateway, i.e., the arduino, interconnected to the internet via ESP8266 which is monitored by an IP address, uploaded in thingspeak over the internet.

The major benefit of uploading the monitored data to the thingspeak is that, it gives the real time physiological condition of the patient, by monitoring the parameters round the clock, along with the values with précised date and time. The major advantage being that the Doctors can evaluate the patient at any time, and the nurses and the in-charge person can be alert by anytime. The thingspeak is also available as an android application, THINGVIEW, which can be accessed by a particular thingspeak account that can be viewed with its API key and it makes it easier for the individual to monitor the subject.

\section{Literature review}

A trip stress monitoring method and device was designed for drivers, where many drivers feel extreme stress when they are stuck in traffic, get cut off by other drivers, and encounter rude drivers, has described a trip stress monitoring device, where the device uses interface of many devices, which are further transmitted over internet by the use of geo-location point, where in the device monitors continuously, the physiological parameters of the driver, displaying a map, where the stored GPS location graphically forms a travel route. [1] The mobile computing device and the physiological parameter monitoring device may be incorporated into one integrated device within a single housing.

A real time patient monitoring system was implemented,[2] where it was used in Intensive Care Units(ICU), along with the internet interfacing system. The system focused on negligence cases in the ICU, where many patients lost lives. This paper states that their proposed model is intelligent in a way that it can monitor the critical conditions of emergency, and can provide push notification to doctors/nurses in that system as well as in charge person of the system. [3]Vital signs are the 4 to 5 most important parameters that need to be monitored to check the patient's life sustaining parameters. These measurements are helpful for a physician to analyze the better physiological parameters specifically, Body temperature, Blood pressure, Pulse rate, Breathing rate, Along with the ECG (with the emergency cases of the ICU). These systems are seen to be used the basic of all devices for monitoring. With the usage of thermometer for temperature, sphygmomanometer for blood pressure, watch or a stethoscope to monitor, pulse.

[4] Presented a prototype system for remote patient monitoring, to fill the gap in monitoring a patient's vital signs between ICU and the actual hospital discharge. It prevents relapses, since; it allows the medical personnel to timely and accurately evaluate the ward patients. Low-power profile sensors that communicate wirelessly with a Gateway type device were implemented, using the Raspberry 
PI B+ board. IoT based medical applications were carried by calling the RESTful API.

In [5], Using IoT, they proposed a disease prediction and monitoring system for stroke patients. The system implemented a microcontroller connected to various wearable sensors and cloud, in which the Input values were collected using sensors and sent to the cloud for storage from where the alert messages get generated.

\section{Methodology}

The pulse rate sensor and the DHT11 are interfaced with an arduino, which is connected with an ESP8266. The arduino transmits the acquired output to thingspeak, which plots the real time data. The basic functional block diagram of the system is explained below in Fig

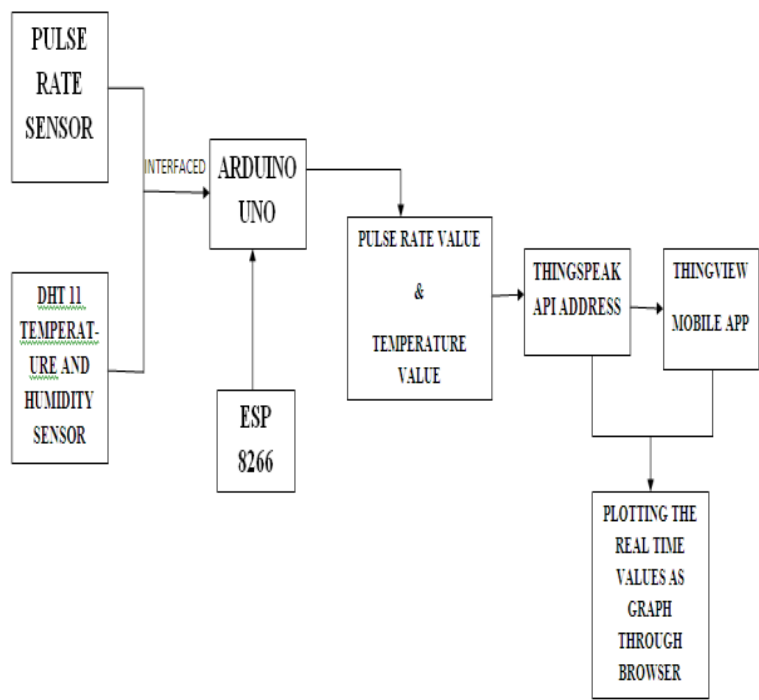

Fig. 1: Block Diagram of the System.

The connections are given to the system as shown below in Figure (2).

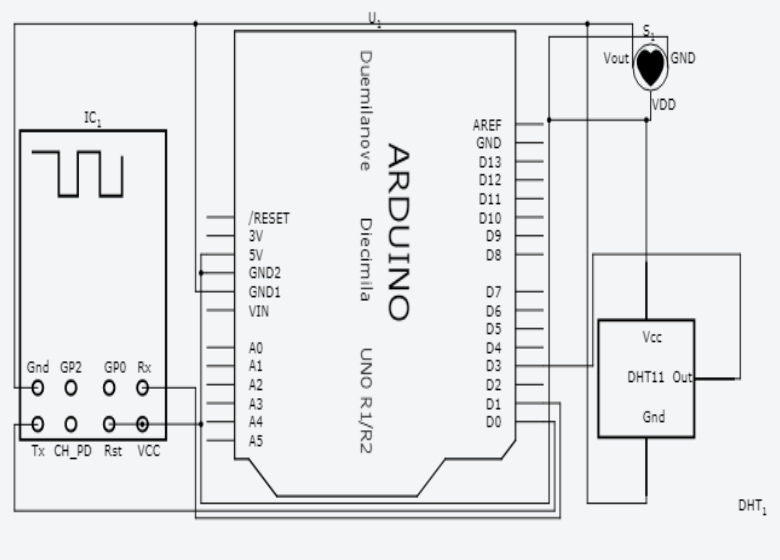

Fig. 2: Circuit Diagram of the System.

Our proposed system consists of the following components

\subsection{Arduino (ATMEL 328p)}

The hardware used in arduino is the Arduino uno, along with a software programmed as simplified C++. Modern applications of arduino are combined with Microcontrollers for various functions through electronic devices as well as functions through internet. An arduino reads information from the given input devices like sensors and other pontentiometric devices which can be further connected to various systems such as LEDs, etc.,

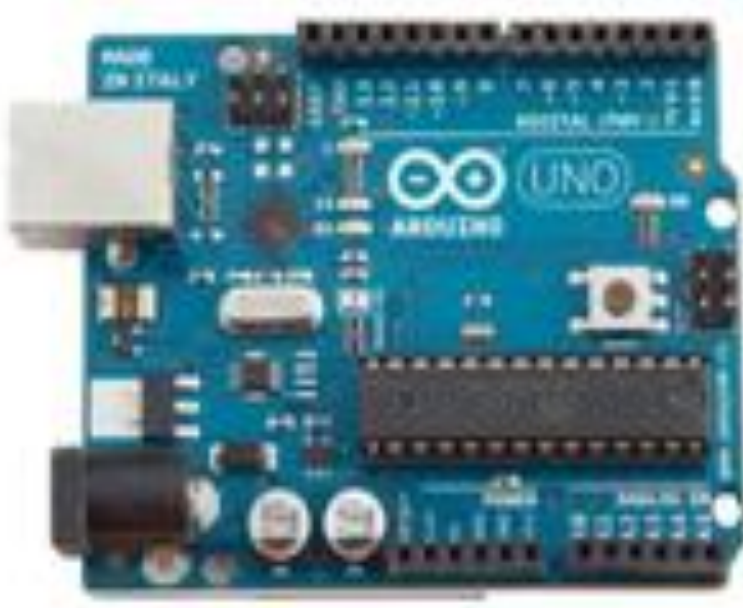

Fig. 3: Arduino UNO.

\subsection{ESP8266}

The ESP8266 is of type 32 bit Microcontroller which holds $64 \mathrm{~KB}$ of instructions and $96 \mathrm{~KB}$ of data. It is a low-cost WiFi microchip provided with an IP and microcontroller capability. It is capable of controlling microcontrollers to connect to a Wi-Fi network. External QSPI flash of up to $16 \mathrm{MB}$ is supported. ARDUINO - A C++ based firmware, this core enables the ESP8266 CPU and its Wi-Fi. The ESP8266 Arduino Core is available through Git Hub. It is an open source basic interpreter specifically designed and developed for internet of things (Iot). The ESP connects to the private API key of the thing speak cloud. In this paper the ESP 8266 connects to the private channel of thing speak cloud via private individual API keys and the data transmitted from ESP is continuously plotted and monitored.

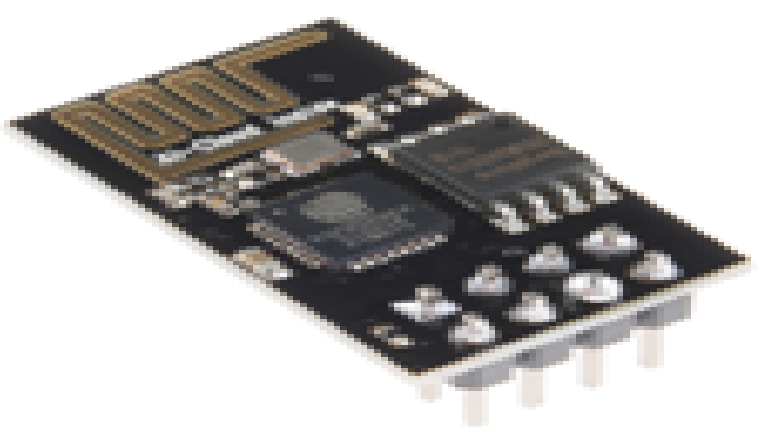

Fig. 4: ESP 8266

\subsection{Pulse rate sensor}

Pulse is the sound produced by the heart valves, with the contaction and relaxation of the heart while the location of the blood flow changes over points. Usually the pulse rate can be sensed physically, through any vein just under the skin by placing one's fingers. The beats produced by the heart are calculated in terms of Beats Per Minute(BPM). But when it comes to the usage of sensors, the pulse rate sensor works on the principle of Photoplethysmography. Where the optical depth determines blood flow. The change in volume of blood flow through any body organ is measured, that causes a change in intensity of light through that place. The most important part of measurement is the timing of the pulse while being monitored.

The pulse rate sensor here is INVNT-11, with an operating voltage of $3.3 \mathrm{~V}$ to $5 \mathrm{~V}$, limiting a current of $4 \mathrm{~mA}$. The system is connected to an indicator LED. Hardware connections of our sensor: Signal(S) to $\mathrm{Ao}, \mathrm{Vcc}(+)$ to $5 \mathrm{~V}$, and ground(-) to GND. 


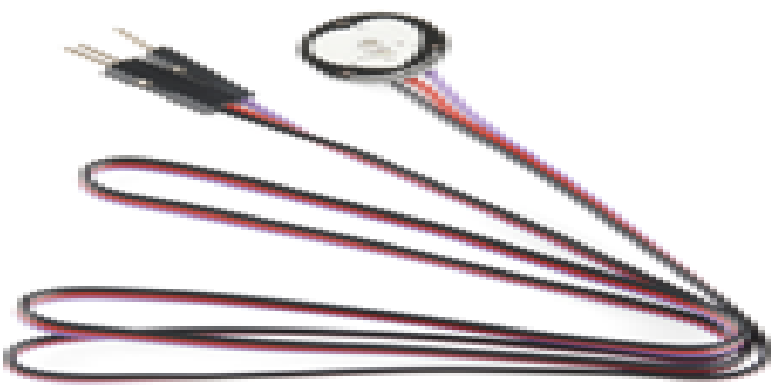

Fig. 5: Pulse Rate Sensor.

\subsection{ECG Sensor}

An ECG displays the voltage between pairs of the chest electrodes. Electrodes placed on the heart measure the electrical activity of different parts of the heart muscle. The lead system used here is 3-lead system, connected via an arduino, plotted and checked on the serial monitor.

\subsection{Temperature sensor (DHT11)}

One of the easiest paramters to monitor in the body is the temperature. Maintainence of temperature is mandatory, as it results in many body changes over time. One of the most important conditons, to mainatin the body temperature is the process of wound healing. Hence,body temperature should be monitored overtime.

Temperature sensor used in this paper, the DHT11 is a basic sensor for the measurement of temperature and humidity, with arduino interfacing. It has $22.0 \mathrm{~mm}$ X $20.5 \mathrm{~mm}$ X $1.6 \mathrm{~mm}$ of PCB size, with a voltage common for both working and operating being 3.3 or $5 \mathrm{~V}$ DC. The resolution is 8 bit for temperature and the same for humidity. Temperature range is $0-50{ }^{\circ} \mathrm{C}$ error of $\pm 2{ }^{\circ} \mathrm{C}$.

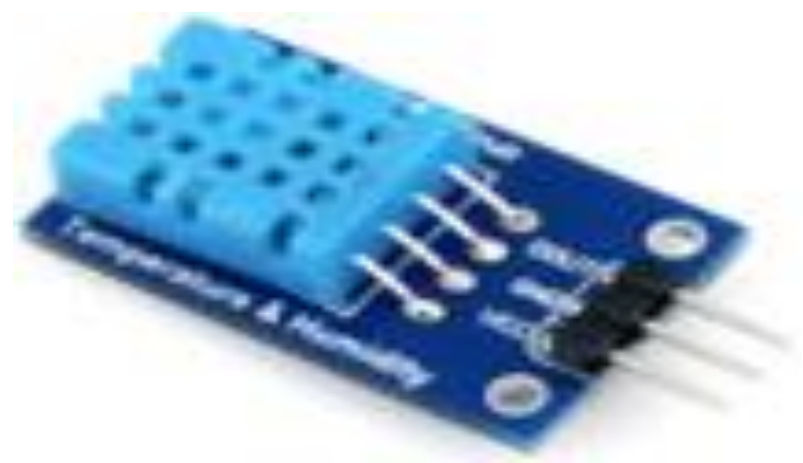

Fig. 6: Dht11 Sensor.

\subsection{Respiration rate transducer}

A piezoelectric device is present that linearly responds to changes in length. Hence, it measures the change in length of the abdominal or thoracic regions while a person is under respiration. Breathing strength, inhaling rate and exhalation rate result in the changes, which are used to monitor the respiration.

\subsection{Thing speak}

It is an open-source Internet of things, and API to store and retrieve data from things that use HTTPS, where a user's account is used to track and monitor the considering data, via IoT. An API key (Application Programming Interface) is a code for calling an application programming interface (API)to pass through computer programs to identify the calling program, its developer, or its user to the Web site.

\section{Results \& discussion}

The pulse rate sensor is interfaced with arduino micro controller. The pulse rate and temperature waveforms are monitored in the arduino uno serial plotter. The pulse rate and temperature rate values of the subject are monitored and transmitted to the thingspeak cloud using ESP8266 IoT device. The Private Channel in the Thing speak website, which gives result and output in the form of a graph, which can be viewed by the Doctor/Caretaker continuously when and where required. Previously, many researchers have transmitted these signals, but used various other devices, such as the Bluetooth, Zigbee, Thinger.io, RaspberryPi, etc,

At first, the pulse rate sensor was held in place, and the signal output was viewed as a graph on the arduino program and then viewed real time on the thingspeak channel. Figure (7) and Figure(8) describe the outputs of app view and website view of the pulse rate sensor respectively. Then the same was repeated using DHT11 sensor and real time view was seen on the thingspeak channel. Figure (9), Figure (10), and Figure (11),describe the outputs of DHT11 temperature, humidity and thingview app measures respectively. The resultant outputs of temperature, humidity and pulse rate, on serial monitor respectively are shown in $\mathrm{Fig}(13)$ and $\mathrm{Fig}(12)$ respectively.

The overall view of the parameters and their outputs with respect to time are shown precisely in Table1. Where the Min and Max pulse rate values should be 72 and 100.1 respectively, and following the Min and Max for temperature to be 30.0 and 100 respectively. Same repeats for humidity to be 55.0 and 100.1 respectively.
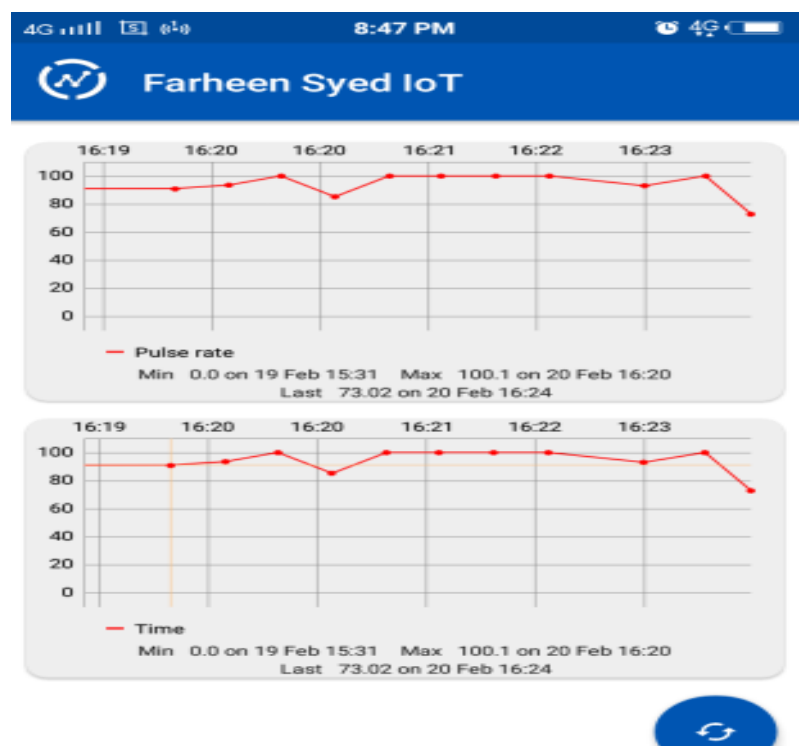

Ad closed by Google

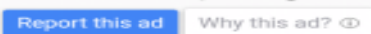

Fig.7: The Resultant Output of the Pulse Rate on Thingview APP

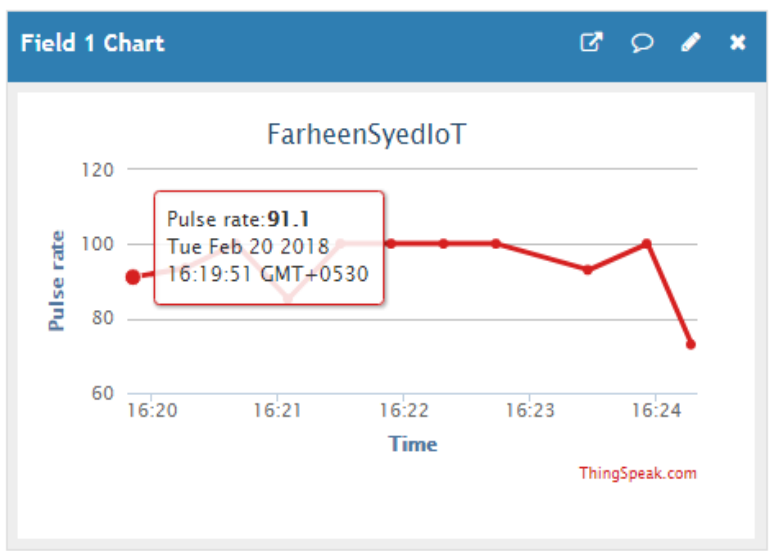

Fig.8: the Resultant Output of Pulse Rate with Respect to Time on Thing Speak Website. 


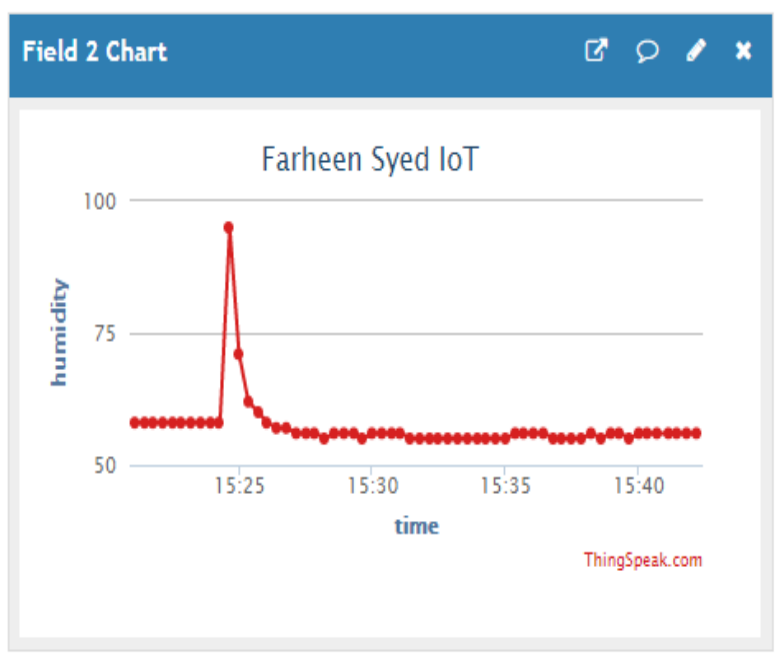

Fig.9: The Resultant Output of Humidity with Respect to Time.

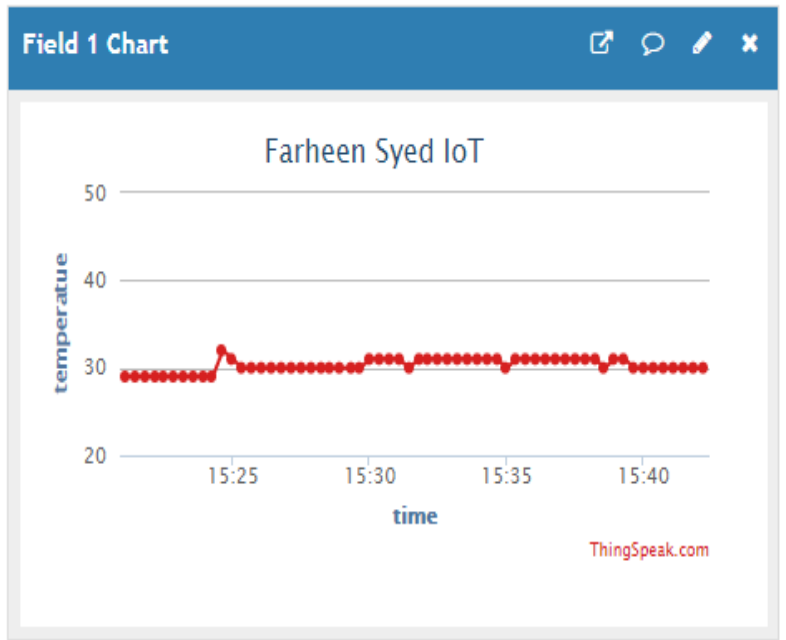

Fig. 10: The Resultant Output of Temperature, with Respect to Time.
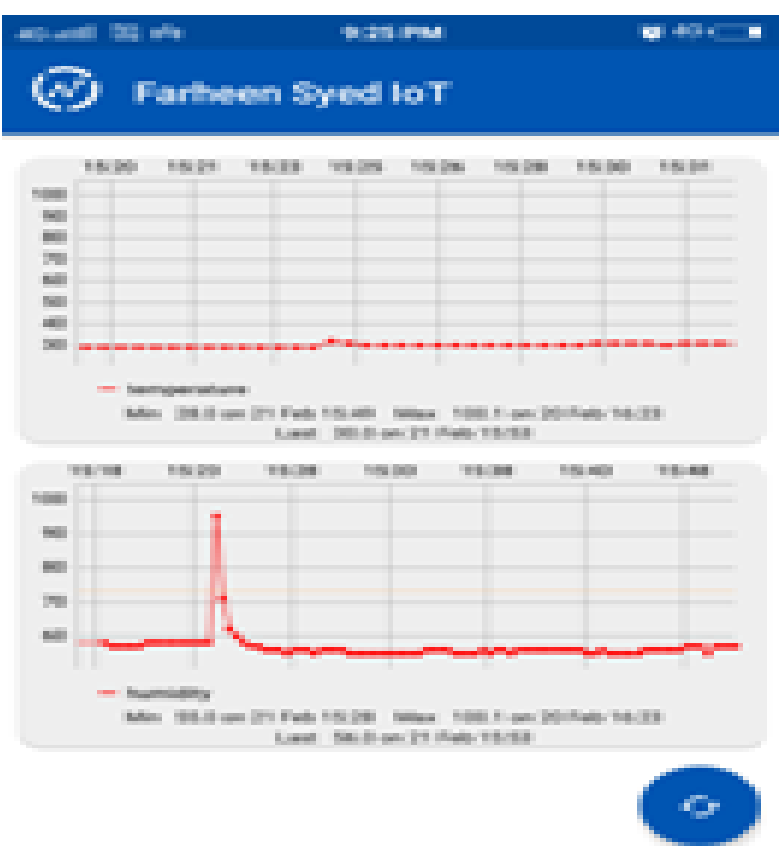

Fig.11: The Resultant output of Temperature and Humidity, on the Thing View APP.

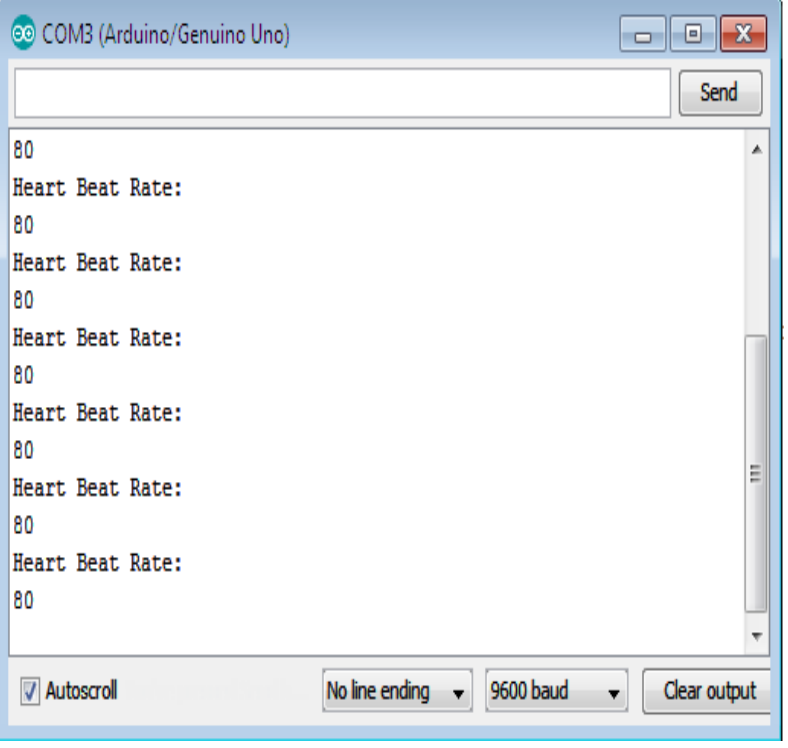

Fig.12: The Resultant Output of Pulse Rate on Serial Monitor

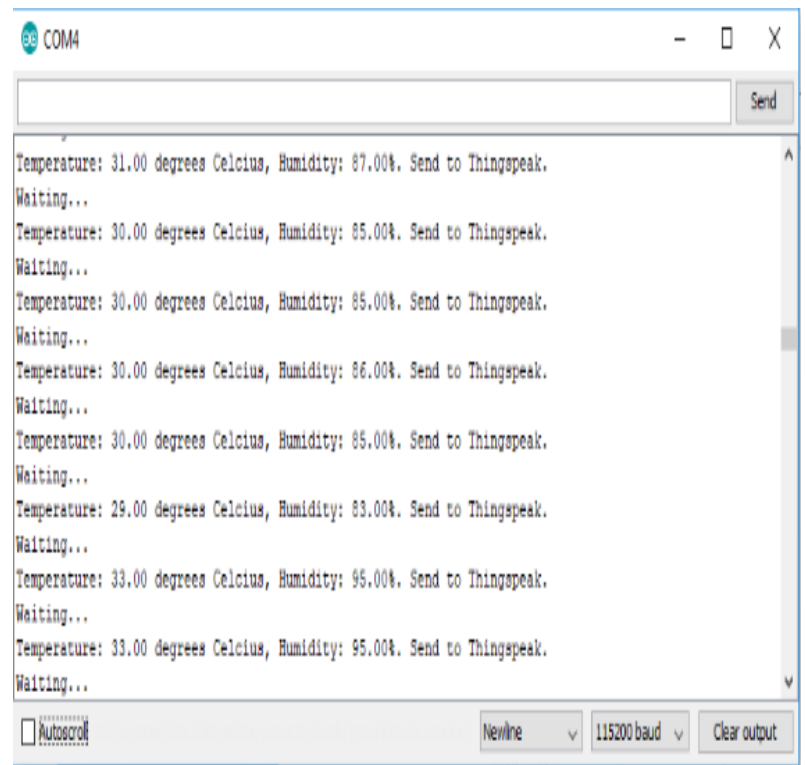

Fig.13: The Resultant Output of Temperature and Humidity on Serial Monitor.

Table 1: Obtained Values of Parameters with Specified Date and Time

\begin{tabular}{llll}
\hline Parameter & Subject & Plot time & Values \\
\hline Heart Rate & Patient 1 & 15:31, Feb 20,Tue,2018 & 73.02 \\
Temperature rate & Patient 1 & 15:53 Feb 21,Wed,2018 & 30.0 \\
Humidity & Patient 1 & 15:53, Feb 21,Wed,2018 & 56 \\
\hline
\end{tabular}

In this paper, we aimed at two basic parameters, namely the Pulse rate monitoring and the Temperature sensing. But the ultimate proposal of this paper is to sense and transmit, all the parameters of the patient monitoring system, with addition to ECG sensors, saturation rate of oxygen, Non Invasive Blood Pressure (NIBP). All these parameters, can be sensed, interfaced with an arduino, transmitted over an IoT device, ESP8266. Which, thereby connects to the thing speak IP address. There will be a rapid development of technology, and hence, health care is one of the aspect that needs to be considered and must be developed over time and with the growth of technology. Hence, the essential parameters of monitoring should be made easy to connect an individual to the doctor. Therefore, development of such systems using IoT is essential.

\section{References}

[1] Josh WEI, Wilmette, IL (US); Parin, PATEL, Mountain View, CA (US); Ida WAHLQUIST, Mountain View, CA (US), Stress and Heart Rate Trip Monitoring System and Method. 
[2] Mohammad Salah Uddin Jannat Binta Alam1, and Suraiya Banu2, “A. Ruberti” Real Time Patient Monitoring System based on Internet of Things.

[3] En.wikipedia.org/wiki/Vital signs.

[4] Alexandru Archip*, Nicolae Botezatu*, Elena S, erban*, Paul-Corneliu Herghelegiu * and Andrei Zal`a $†$, An IoT Based System for Remote Patient Monitoring.

[5] Ani R1, Krishna S1, Anju N1, Sona Aslam M1, O.S Deepa2, IoT Based Patient Monitoring and Diagnostic. Prediction Tool using Ensemble Classifier.

[6] Whitmore, A. Agarwal, and L. Xu, "The Tnternet of Things- A Survey of Topics and Trends," Information Systems Frontiers, vol. 17, no. 2, pp. 261-274, Apr. 2015.

[7] R.Kumar, Dr.M.Pallikonda Rajasekaran, AN IOT BASED PATIENT MONITORING SYSTEM USING RASPBERRY PI

[8] GSMA, "Gsma: The impact of the internet of things - the connected Home,” KRC RESEARCH, Tech. Rep., 2012.

[9] G. Kortuem, F. Kawsar, D. Fitton, and V. Sundramoorthy, “'Smart objects

As building blocks for the Internet of Things," IEEE Internet Comput., vol. 14, no. 1, pp. 44_51, Jan. /Feb. 2010.

[10] K. Romer, B. Ostermaier, F. Mattern, M. Fahrmair, and W. Kellerer, "Real-time search for real-world entities: A survey," Proc. IEEE, vol. 98, No. 11, pp. 1887_1902, Nov. 2010.

[11] D. Guinard, V. Trifa, and E. Wilde, “A resource oriented architecture For the Web of Things," in Proc. Internet Things (IOT), Nov. /Dec. 2010.

[12] Unnati Dhanaliya, Anupam Devani. Implementation of E-Health Care System using Web Services and Cloud Computing.

[13] S. Li, L. D. Xu, and S. Zhao, "The Internet of Things: A Survey," Information Systems Frontiers, vol. 17, no. 2, pp. 243-259, 2015.

[14] Chanchal Raj, 1 Chaman Jain2 and Wasim Arif3, HEMAN: Health Monitoring and Nous, An IoT based e-Health Care System for Remote Telemedicine. 